\title{
Coexistence of Congenital Adrenal Hyperplasia and Autoimmune Addison's Disease
}

\section{OPEN ACCESS}

Edited by: Sandro Loche,

Ospedale Microcitemico, Italy

Reviewed by:

Alberto Falorni,

University of Perugia, Italy

Luigi R. Garibaldi,

University of Pittsburgh, United States

*Correspondence:

Sigrid Aslaksen

sigrid.aslaksen@uib.no

Specialty section

This article was submitted to

Pediatric Endocrinology,

a section of the journal

Frontiers in Endocrinology

Received: 10 July 2019 Accepted: 06 September 2019 Published: 27 September 2019

Citation:

Aslaksen S, Methlie P, Vigeland MD, Jøssang $D E$, Wolff $A B$, Sheng $Y$,

Oftedal BE, Skinningsrud $B$,

Undlien DE, Selmer KK, Husebye ES and Bratland E (2019) Coexistence of Congenital Adrenal Hyperplasia and Autoimmune Addison's Disease.

Front. Endocrinol. 10:648. doi: $10.3389 /$ fendo.2019.00648

\begin{abstract}
Sigrid Aslaksen 1,2*, Paal Methlie 1,2,3, Magnus D. Vigeland 4,5, Dag E. Jøssang ${ }^{6}$, Anette B. Wolff ${ }^{1,2}$, Ying Sheng ${ }^{5}$, Bergithe E. Oftedal ${ }^{1,2}$, Beate Skinningsrud ${ }^{5}$, Dag E. Undlien ${ }^{4,5}$, Kaja K. Selmer ${ }^{7,8}$, Eystein S. Husebye ${ }^{1,2,3}$ and Eirik Bratland ${ }^{1,2}$

${ }^{1}$ Department of Clinical Science, University of Bergen, Bergen, Norway, ${ }^{2}$ K. G. Jebsen Center for Autoimmune Diseases, University of Bergen, Bergen, Norway, ${ }^{3}$ Department of Medicine, Haukeland University Hospital, Bergen, Norway, ${ }^{4}$ Institute of Clinical Medicine, University of Oslo, Oslo, Nonway, ${ }^{5}$ Department of Medical Genetics, Oslo University Hospital, Oslo, Norway, ${ }^{6}$ Department of Radiology, Haukeland University Hospital, Bergen, Norway, ${ }^{7}$ Division of Clinical Neuroscience, Department of Research and Development, Oslo University Hospital, University of Oslo, Oslo, Norway, ${ }^{8}$ National Centre for Epilepsy, Oslo University Hospital, Oslo, Norway
\end{abstract}

Background: Underlying causes of adrenal insufficiency include congenital adrenal hyperplasia $(\mathrm{CAH})$ and autoimmune adrenocortical destruction leading to autoimmune Addison's disease (AAD). Here, we report a patient with a homozygous stop-gain

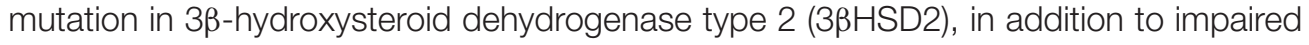
steroidogenesis due to AAD.

Case Report: Whole exome sequencing revealed an extremely rare homozygous nonsense mutation in exon 2 of the HSD3B2 gene, leading to a premature stop codon (NM_000198.3: c.15C>A, p.Cys5Ter) in a patient with AAD and premature ovarian insufficiency. Scrutiny of old medical records revealed that the patient was initially diagnosed with $\mathrm{CAH}$ with hyperandrogenism and severe salt-wasting shortly after birth. However, the current steroid profile show complete adrenal insufficiency including low production of pregnenolone, dehydroepiandrosterone (DHEA) and DHEA sulfate (DHEA-S), without signs of overtreatment with steroids.

Conclusion: To the best of our knowledge, this is the first description of autoimmune adrenalitis in a patient with $3 \beta \mathrm{HSD} 2$ deficiency and suggests a possible association between $A A D$ and inborn errors of the steroidogenesis.

Keywords: adrenal insufficiency, congenital adrenal hyperplasia, 3 $\beta$-hydroxysteroid dehydrogenase type 2 deficiency, autoimmune adrenalitis, autoimmune Addison's disease

\section{INTRODUCTION}

$3 \beta$-hydroxysteroid dehydrogenase type 2 ( $3 \beta \mathrm{HSD} 2)$ catalyzes the conversion of $\Delta 5$-steroids into $\Delta 4$-steroids (Figure 1). Deficiency of $3 \beta \mathrm{HSD} 2$ causes a rare autosomal recessive form of congenital adrenal hyperplasia $(\mathrm{CAH})$ characterized by high levels of pregnenolone, 17-hydroxypregnenolone, dehydroepiandrosterone (DHEA), DHEA sulfate (DHEA-S), and androstenediol, and lack of

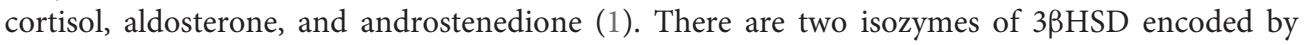
$H S D 3 B 1$ and $H S D 3 B 2.3 \beta H S D 2$ is expressed in the gonads and the adrenal cortex, whereas $3 \beta H S D 1$ is expressed in peripheral tissues, converting circulating DHEA to testosterone (1). 3BHSD2 


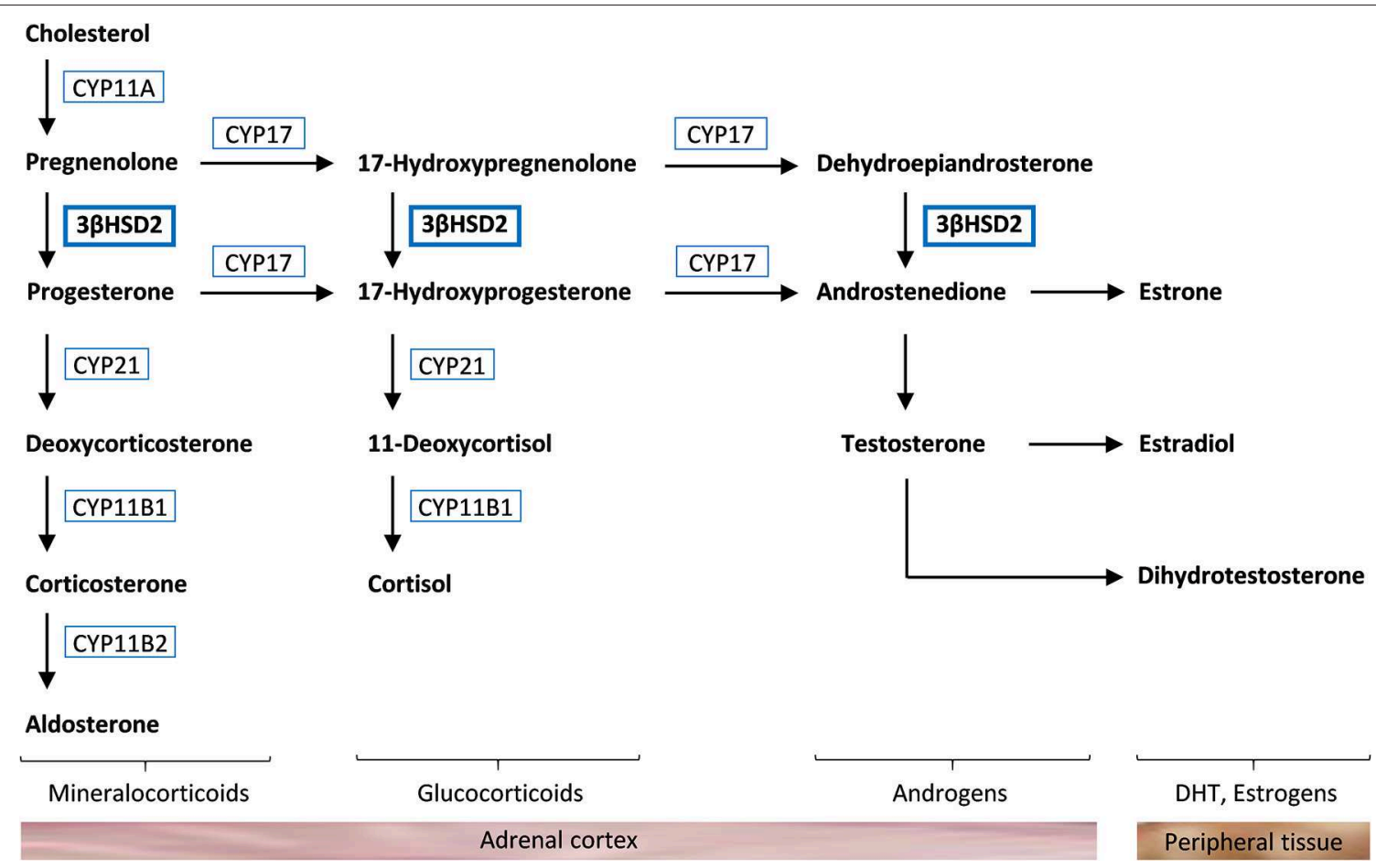

FIGURE 1 | Steroidogenesis in the adrenal cortex. Cholesterol is converted to aldosterone, cortisol, and androgens through different pathways that require specific

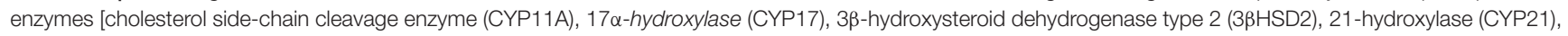
11ß-hydroxylase (CYP11B1), and aldosterone synthase (CYP11B2)]. Androstenedione and testosterone are further converted to dihydrotestosterone (DHT) and estrogens in peripheral tissue.

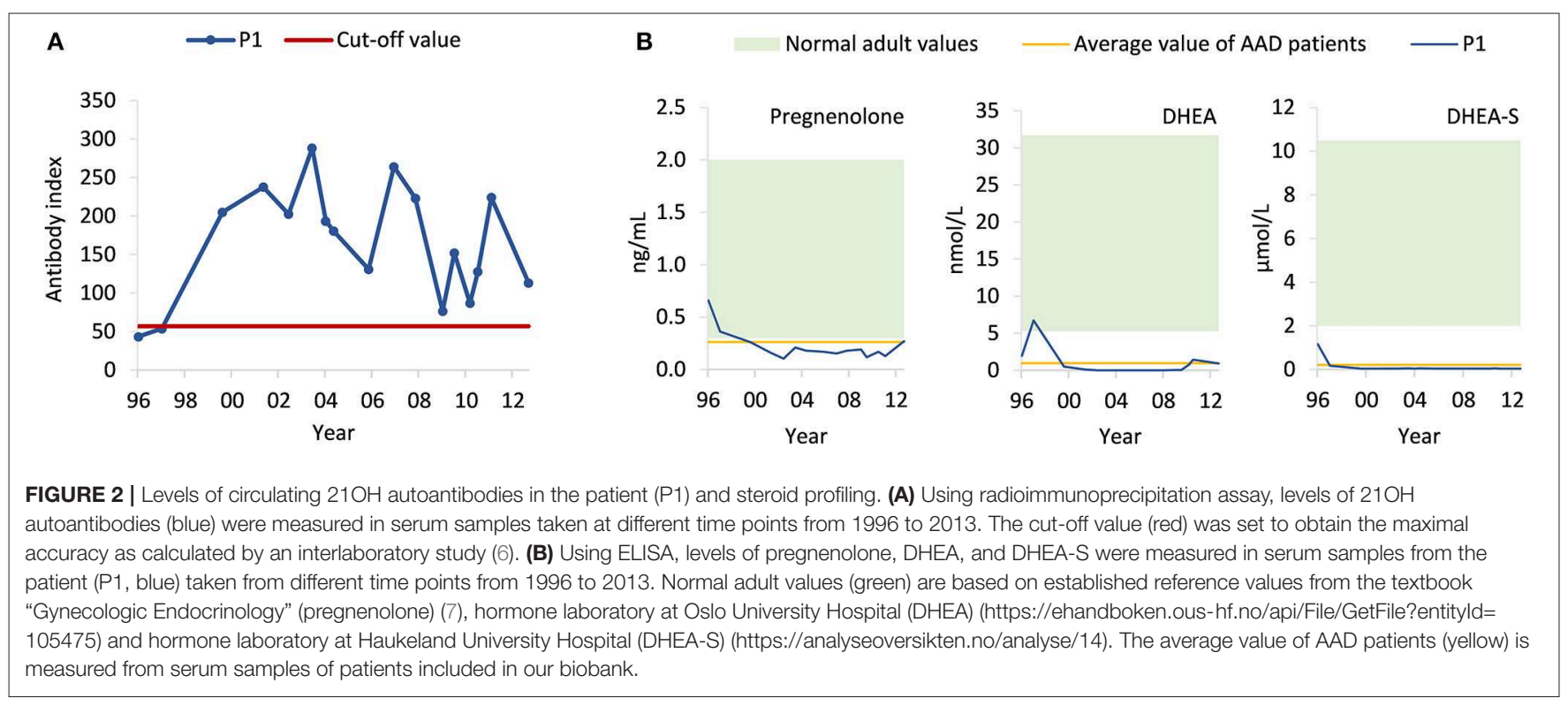

deficiency can therefore cause relatively high levels of testosterone in females, whereas it cannot compensate for the absence of adrenal and gonadal synthesis of testosterone in males. This causes ambiguous genitalia in males, whereas female newborns exhibit mild virilization or normal sexual differentiation, and may remain undiagnosed until a salt-wasting crisis occurs (1-3).
Adrenal insufficiency can also be due to autoimmune adrenalitis, or autoimmune Addison's disease (AAD), characterized by an immunological attack of the adrenal cortex leading to decreased production of cortisol and aldosterone (4). The self-antigen 21-hydroxylase $(21 \mathrm{OH})$ is the dominant target of adrenal autoantibodies and autoreactive $\mathrm{T}$ cells (4). Therefore, autoantibodies against $21 \mathrm{OH}$ and 
low serum cortisol levels are important diagnostic markers for AAD.

To the best of our knowledge, we here report the first patient affected by both inborn $3 \beta \mathrm{HSD} 2$ deficiency and acquired AAD.

\section{CASE REPORT}

A whole-exome sequencing study involving 142 AAD patients (5) revealed a patient with a rare homozygous mutation in exon 2 of HSD3B2 [frequency $\sim 0.00003$ in the Genome Aggregation Database (gnomAD)] at nucleotide position 15 (NM_000198.3:c.15C>A), resulting in the exchange of the cysteine codon to a premature stop codon (p.Cys5Ter). Subsequent screening of the Norwegian Addison Registry identified the patient harboring the mutation, a 55-yearold female with AAD, premature ovarian insufficiency and vitamin B12 deficiency, accompanied by $21 \mathrm{OH}$ - and parietal cell autoantibodies (Figure 2A). Notably, she did not carry any of the major histocompatibility complex (MHC) alleles conferring high risk to develop AAD (4). She tested negative for other autoantibodies such as anti-thyroid peroxidase, and had normal levels of thyroid stimulating hormone (0.40$4.50 \mathrm{mIU} / \mathrm{L})$. Scrutiny of early medical records showed that

TABLE 1 | Steroid profile of the patient.

\begin{tabular}{|c|c|c|}
\hline Steroids & LLoQ (nM) & Patient serum sample \\
\hline Progesterone & 0.114 & $<$ LLoQ \\
\hline 11-deoxycorticosterone & 0.023 & $<$ LLoQ \\
\hline Corticosterone & 0.114 & $<$ LLoQ \\
\hline Tetrahydrocorticosterone & 0.114 & $<$ LLoQ \\
\hline 18-hydroxycorticosterone & 0.069 & $<$ LLoQ \\
\hline Aldosterone & 0.0023 & $<$ LLoQ \\
\hline Tetrahydroaldosterone & 0.062 & $<\mathrm{LLOQ}$ \\
\hline 17-hydroksyprogesterone & 0.114 & $<$ LLoQ \\
\hline 11-deoxycortisol & 0.114 & $<$ LLoQ \\
\hline 21-deoxycortisol & 0.023 & $<\mathrm{LLOQ}$ \\
\hline Cortisol & 0.914 & $<$ LLoQ \\
\hline Tetrahydrocortisol & 0.114 & 0.308 \\
\hline $5 \alpha$-tetrahydrocortisol & 0.114 & 0.187 \\
\hline 18-hydroxycortisol & 0.046 & $<$ LLoQ \\
\hline 18-oxocortisol & 0.046 & $<$ LLoQ \\
\hline Cortisone & 0.914 & $<\mathrm{LLOQ}$ \\
\hline Tetrahydrocortisone & 0.343 & $<$ LLoQ \\
\hline $5 \alpha$-tetrahydrocortisone & 0.343 & $<$ LLoQ \\
\hline Dehydroepiandrosterone & 0.617 & $<$ LLoQ \\
\hline Dehydroepiandrosterone sulfate & 22.862 & $<$ LLoQ \\
\hline Androstenedione & 0.023 & $<$ LLoQ \\
\hline Testosterone & 0.023 & 0.066 \\
\hline Dihydrotestosterone & 0.023 & $<$ LLoQ \\
\hline Epitestosterone & 0.023 & $<$ LLoQ \\
\hline
\end{tabular}

Overview of the levels of 24 analytes in a fasting serum sample from the patient, including the Lower Limit of Quantification Levels (LLOQ) for each analyte. The analysis was performed using LC-MS/MS.

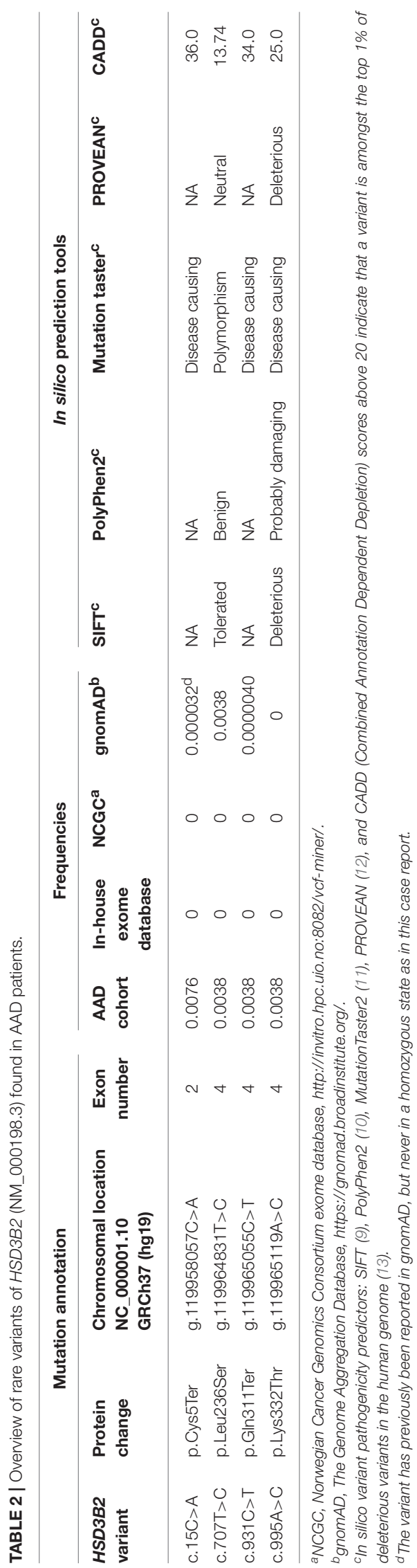


she exhibited hyperpigmentation of genitalia and clitoris hypertrophy already at birth. One week of age, she started to vomit and developed hyponatremia (127 mmol/L) and hyperkalemia $(6.1 \mathrm{mmol} / \mathrm{L})$. Elevated levels of 17 -ketosteroids were detected. She was therefore diagnosed with $\mathrm{CAH}$ and supplemented with cortisone acetate and eventually fludrocortisone. Her sister had also been diagnosed with $\mathrm{CAH}$, but unfortunately died in an adrenal crisis at 2 years of age.

Given these conflicting findings, computer tomography (CT) and magnetic resonance (MR) scans, taken from age 43 to 51 , were re-evaluated and the adrenals were found to be in the lower range of normal thickness $2-3 \mathrm{~mm}$, indicating adrenocortical atrophy rather than hyperplasia as would be expected in case of isolated CAH. We then obtained a steroid profile by liquid chromatography tandem mass spectroscopy (LC-MS/MS) of a serum sample taken after an overnight medication fast (Table 1). Levels of all mineralocorticoids, and most glucocorticoids and androgens were below the detection limit, except for tetrahydrocortisol $(0.308 \mathrm{nmol} / \mathrm{L})$, $5 \alpha$-tetrahydrocortisol $(0.187 \mathrm{nmol} / \mathrm{L})$, and testosterone (0.066 nmol/L). Total pregnenolone, DHEA and DHEA-S levels were measured by enzyme-linked immunosorbent assay (ELISA) from previously collected serum samples spanning the years 1996-2013 (Figure 2B). Normal levels of pregnenolone and DHEA were found in samples from the first 2 years, but then levels decreased toward the subnormal levels generally seen in AAD patients. DHEA-S was also below the normal range, typical of AAD patients, at all time points. ACTH was measured at several time points, revealing elevated levels on multiple occasions. The highest levels were observed in 2011 at $130 \mathrm{pmol} / \mathrm{L}$ (normal range 2.0-11.6 $\mathrm{pmol} / \mathrm{L}$ ). Importantly, we detected no subsequent increase in pregnenolone, DHEA, and DHEA-S in spite of elevated ACTH levels (Figure 2B).

She is currently, at age 57, treated with $20 \mathrm{mg}$ hydrocortisone (Plenadren $^{\mathrm{TM}}$ ) and $100 \mu \mathrm{g}$ fludrocortisone, and does not have suppressed ACTH-values.

\section{DISCUSSION}

This is the first report of a patient with primary adrenal insufficiency due to both $3 \beta \mathrm{HSD} 2$ deficiency and AAD. At inclusion in the national Addison registry, she was classified as having AAD with vitamin B12 deficiency, and 21OHand parietal cell autoantibodies. However, genetic screening and scrutiny of old records revealed a rare form of $\mathrm{CAH}$ due to a stop-gain mutation in HSD3B2. The presence of small sized adrenal glands instead of hyperplastic glands, normally seen in $\mathrm{CAH}$, and no overproduction of $\Delta 5$-steroids following elevated ACTH levels, suggest that the adrenal cortex is not functioning. Presence of tetrahydrocortisol and $5 \alpha$-tetrahydrocortisol is consistent with her ongoing replacement therapy which includes hydrocortisone. The low, but detectable, level of testosterone may be due to conversion of DHEA by $3 \beta H S D 1$ in the periphery. Previously measureable pregnenolone levels suggest that the autoimmune destruction of the adrenal cortex commenced sometimes in the 1990-ties.

According to literature, we could only find one reported case of CAH occurring together with complete adrenal cortex insufficiency suspected to be autoimmune adrenalitis. This patient, however, had neither $21 \mathrm{OH}$ autoantibodies, nor MHC risk alleles, but was positive for autoantibodies against $17 \alpha$ hydroxylase (8). Therefore, we speculate there might be other rare unreported cases of autoimmune adrenalitis due to early diagnosis of $\mathrm{CAH}$, masking the clinical symptoms of AAD. Interestingly, several other AAD patients included in our exome sequencing analysis carry rare heterozygous non-synonymous variants in HSD3B2 (Table 2). Although it appears that family members of $\mathrm{CAH}$ patients, carrying heterozygous HSD3B2 mutations, maintain normal 3 $\beta \mathrm{HSD} 2$ activity in vivo (14), genetic variations in $H S D 3 B 2$ are associated with other conditions such as idiopathic hypospadias and prostate cancer $(15,16)$. Therefore, both subtle molecular abnormalities and deleterious mutations in HSD3B2 could have biological consequences, and may play a role in the pathogenesis of the immune-mediated adrenocortical destruction in AAD.

\section{DATA AVAILABILITY STATEMENT}

This manuscript contains previously unpublished data. The name of the repository and accession number(s) are not available.

\section{ETHICS STATEMENT}

The studies involving human participants were reviewed and approved by Regional Committee for Medical and Health Ethics. The patients/participants provided their written informed consent to participate in this study. Written informed consent was obtained from the individual(s) for the publication of any potentially identifiable images or data included in this article.

\section{AUTHOR CONTRIBUTIONS}

All authors listed have made a substantial, direct and intellectual contribution to the work, and approved it for publication.

\section{FUNDING}

This work was supported by the Novo Nordisk Foundation (grant number NNF14OC0011005); and the Research Council of Norway (grant numbers 250030, 262677).

\section{ACKNOWLEDGMENTS}

We thank the patients participating in our research, and Nina Henne, Elisabeth Halvorsen, Elin Theodorsen, and Hajirah Muneer for great technical assistance. 


\section{REFERENCES}

1. Al Alawi AM, Nordenstrom A, Falhammar H. Clinical perspectives in congenital adrenal hyperplasia due to $3 \beta$-hydroxysteroid dehydrogenase type 2 deficiency. Endocrine. (2019) 63:407-21. doi: 10.1007/s12020-01 8-01835-3

2. Alos N, Moisan A-M, Ward L, Desrochers M, Legault L, Leboeuf G, et al. A novel A10E homozygous mutation in the HSD3B2 gene causing severe salt-wasting $3 \beta$-hydroxysteroid dehydrogenase deficiency in 46,XX and 46,XY French-Canadians: evaluation of gonadal function after puberty*. J Clin Endocrinol Metab. (2000) 85:1968-74. doi: 10.1210/jc.85.5.1968

3. Burckhardt MA, Udhane SS, Marti N, Schnyder I, Tapia C, Nielsen JE, et al. Human $3 \beta$-hydroxysteroid dehydrogenase deficiency seems to affect fertility but may not harbor a tumor risk: lesson from an experiment of nature. Eur J Endocrinol. (2015) 173:K1-2. doi: 10.1530/EJE-15-0599

4. Bratland E, Husebye ES. Cellular immunity and immunopathology in autoimmune Addison's disease. Mol Cell Endocrinol. (2011) 336:180-90. doi: 10.1016/j.mce.2010.12.015

5. Aslaksen S, Wolff AB, Vigeland MD, Breivik L, Sheng Y, Oftedal $\mathrm{BE}$, et al. Identification and characterization of rare Toll-like receptor 3 variants in patients with autoimmune Addison's disease. J Transl Autoimmun. (2019) 2019:100005. doi: 10.1016/j.jtauto.2019. 100005

6. Falorni A, Bini V, Betterle C, Brozzetti A, Castaño L, Fichna M, et al. Determination of 21-hydroxylase autoantibodies: inter-laboratory concordance in the Euradrenal International Serum Exchange Program. Clin Chem Lab Med. (2015) 53:1761-70. doi: 10.1515/cclm-2014-1106

7. Josimovich J. Gynecologic Endocrinology. New York, NY: Springer Science \& Business Media (2013).

8. Reinehr T, Rothermel J, Wegener-Panzer A, Hartmann MF, Wudy SA, Holterhus PM. Vanishing 17-hydroxyprogesterone concentrations in 21-hydroxylase deficiency. Horm Res Paediatr. (2018) 90:138-44. doi: $10.1159 / 000487927$

9. Kumar P, Henikoff S, Ng PC. Predicting the effects of coding non-synonymous variants on protein function using the SIFT algorithm. Nat Protoc. (2009) 4:1073-81. doi: 10.1038/nprot.2009.86
10. Adzhubei I, Jordan DM, Sunyaev SR. Predicting functional effect of human missense mutations using PolyPhen-2. Curr Protoc Hum Genet. (2013) Chapter7:Unit7.20. doi: 10.1002/0471142905.hg0720s76

11. Schwarz JM, Cooper DN, Schuelke M, Seelow D. MutationTaster2: mutation prediction for the deep-sequencing age. Nat Methods. (2014) 11:361-2. doi: $10.1038 /$ nmeth. 2890

12. Choi Y, Sims GE, Murphy S, Miller JR, Chan AP. Predicting the functional effect of amino acid substitutions and indels. PLoS ONE. (2012) 7:e46688. doi: 10.1371/journal.pone.0046688

13. Kircher M, Witten DM, Jain P, O'roak BJ, Cooper GM, Shendure J. A general framework for estimating the relative pathogenicity of human genetic variants. Nat Genet. (2014) 46:310-5. doi: 10.1038/ng.2892

14. Pang S, Carbunaru G, Haider A, Copeland KC, Chang YT, Lutfallah C, et al. Carriers for type II 3 $\beta$-hydroxysteroid dehydrogenase (HSD3B2) deficiency can only be identified by HSD3B2 genotype study and not by hormone test. Clin Endocrinol. (2003) 58:323-31. doi: 10.1046/j.1365-2265.2003.01716.x

15. Codner E, Okuma C, Iñiguez Gn, Boric MAl, Avila A, Johnson MC, et al. Molecular study of the $3 \beta$-hydroxysteroid dehydrogenase gene type II in patients with hypospadias. J Clin Endocrinol Metab. (2004) 89:957-64. doi: $10.1210 /$ jc.2002-020873

16. Neslund-Dudas C, Bock CH, Monaghan K, Nock NL, Yang JJ, Rundle A, et al. SRD5A2 and HSD3B2 polymorphisms are associated with prostate cancer risk and aggressiveness. Prostate. (2007) 67:1654-63. doi: 10.1002/pros.20625

Conflict of Interest: The authors declare that the research was conducted in the absence of any commercial or financial relationships that could be construed as a potential conflict of interest.

Copyright (c) 2019 Aslaksen, Methlie, Vigeland, Jøssang, Wolff, Sheng, Oftedal, Skinningsrud, Undlien, Selmer, Husebye and Bratland. This is an open-access article distributed under the terms of the Creative Commons Attribution License (CC BY). The use, distribution or reproduction in other forums is permitted, provided the original author(s) and the copyright owner(s) are credited and that the original publication in this journal is cited, in accordance with accepted academic practice. No use, distribution or reproduction is permitted which does not comply with these terms. 